\title{
COVID-19 and chronic kidney disease: an updated overview of reviews
}

\author{
Sara S. Jdiaa ${ }^{1} \cdot$ Razan Mansour $^{2} \cdot$ Abdallah El Alayli $^{2} \cdot$ Archana Gautam $^{2} \cdot$ Preston Thomas $^{3} \cdot$ Reem A. Mustafa $^{2,4}$
}

Received: 30 July 2021 / Accepted: 8 November 2021 / Published online: 11 January 2022

(c) The Author(s) under exclusive licence to Italian Society of Nephrology 2021

\begin{abstract}
Background Coronavirus disease (COVID-19) has resulted in the death of more than 3.5 million people worldwide. While COVID-19 mostly affects the lungs, different comorbidities can have an impact on its outcomes. We performed an overview of reviews to assess the effect of Chronic Kidney Disease (CKD) on contracting COVID-19, hospitalization, mortality, and disease severity.

Methods We searched published and preprint databases. We updated the reviews by searching for primary studies published after August 2020, and prioritized reviews that are most updated and of higher quality using the AMSTAR tool.

Results We included 69 systematic reviews and 66 primary studies. Twenty-eight reviews reported on the prevalence of CKD among patients with COVID-19, which ranged from 0.4 to $49.0 \%$. One systematic review showed an increased risk of hospitalization in patients with CKD and COVID-19 (RR $=1.63,95 \%$ CI 1.03-2.58) (Moderate certainty). Primary studies also showed a statistically significant increase of hospitalization in such patients. Thirty-seven systematic reviews assessed mortality risk in patients with CKD and COVID-19. The pooled estimates from primary studies for mortality in patients with CKD and COVID-19 showed a HR of 1.48 (95\% CI 1.33-1.65) (Moderate certainty), an OR of 1.77 (95\% CI 1.54-2.02) (Moderate certainty) and a RR of 1.6 (95\% CI 0.88-2.92) (Low certainty).

Conclusions Our review highlights the impact of CKD on the poor outcomes of COVID-19, underscoring the importance of identifying strategies to prevent COVID-19 infection among patients with CKD.
\end{abstract}

Reem A. Mustafa

rmustafa@kumc.edu

1 Division of Nephrology, University of Toronto, Toronto, ON, Canada

2 Division of Nephrology and Hypertension, Department of Internal Medicine, Medical Center, University of Kansas, Kansas City, KS, USA

3 School of Medicine, University of Kansas, Kansas City, KS, USA

4 Department of Health Research Methods, Evidence and Impact, McMaster University, Hamilton, ON, Canada 


\section{Graphical abstract}

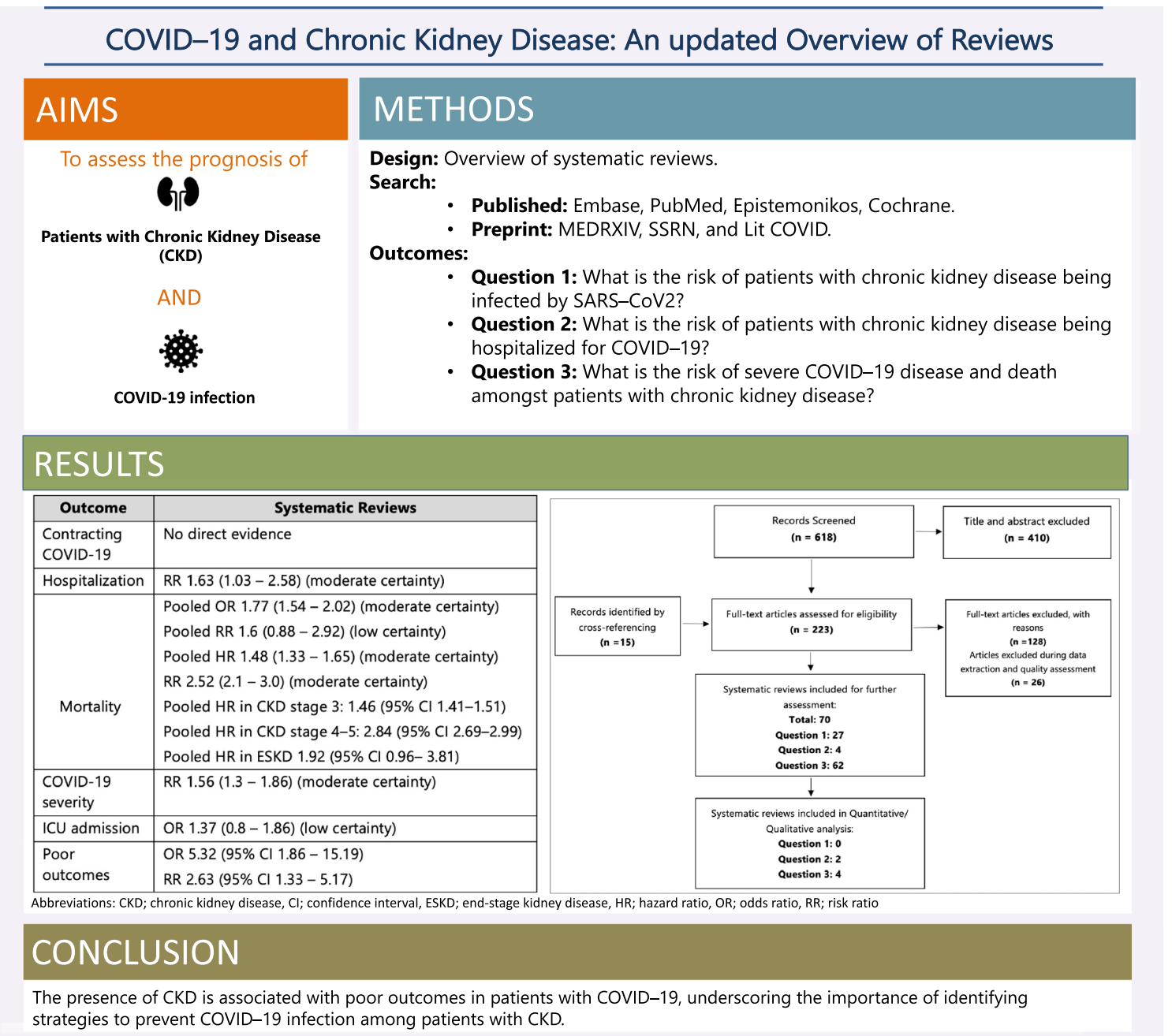

Keywords COVID-19 · SARS-CoV-2 $\cdot$ Chronic kidney disease (CKD) $\cdot$ Mortality $\cdot$ Hospitalization

\section{Introduction}

Since its emergence in December 2019, the severe acute respiratory syndrome coronavirus 2 (SARS-CoV-2) has caused over 240 million confirmed cases and more than 5 million deaths worldwide at the time of writing [1]. The World Health organization (WHO) declared it to be a global pandemic in March 2020. Multiple studies have assessed the association between different comorbidities and coronavirus disease 2019 (COVID-19) outcomes [2, 3]. COVID-19 preferentially affects the lungs with a potential to involve multiple organ systems, including the kidneys.

The global prevalence of chronic kidney disease (CKD) is estimated to be between 9 and $12 \%$ [4]. The incidence of CKD increases with age, and about $38 \%$ of the estimated CKD population is $>65$ years of age [5]. The definition and classification of CKD was established and endorsed by the National Kidney Foundation Kidney Disease Outcomes Quality Initiative and the international Kidney Disease Improving Global Outcomes (KDIGO) guideline group which categorizes CKD into five stages based on the estimated glomerular filtration rate (eGFR) (level) with further sub-classification of stage 5 into dialysis-dependent and dialysis-independent [6]. Advanced CKD is associated with a marked increase in the risk of all-cause mortality and morbidity [7]. Cardiovascular causes are estimated to account for $50 \%$ of the mortality in patients with CKD, while infections are recognized as a leading cause of non-cardiovascular morbidity and mortality in patients with advanced CKD [8-11].

In this updated overview of reviews, we aim to summarize the effect of CKD on different outcomes among patients 
with COVID-19. We reviewed available systematic reviews and large primary studies to assess COVID-19 incidence, severity, risk of hospitalization, and mortality among patients with CKD.

\section{Materials and methods}

The protocol for this overview was published online and is available on PROSPERO (International Prospective Register of Systematic Reviews). The registration number is CRD42021227974. There were no amendments from the pre-specified criteria reported in the protocol throughout the review process. The results are reported according to Preferred Reporting Items for Systematic reviews and MetaAnalyses (PRISMA) guidelines [12].

\section{Inclusion and exclusion criteria}

We started by conducting an overview of systematic reviews that reported COVID-19 outcomes in patients with CKD from January 1, 2020 to January 5, 2021. After searching systematic reviews, we updated the search by identifying primary studies published after August 2020, which was the date of last search in the reviews. We included all published and unpublished studies of any design including retrospective, prospective, and cross-sectional observational studies. The review included studies of adult patients with suspected or confirmed COVID-19 who had CKD. The update focused on primary studies with more than 1000 patients with COVID-19. We excluded systematic reviews and primary studies focusing on children, pregnant women, kidney transplant recipients, and those with acute kidney injury. We prioritized the following PICO questions, which we addressed in the review:

PICO 1: What is the risk of patients with chronic kidney disease being infected by SARS-CoV2?

PICO 2: What is the risk of patients with chronic kidney disease being hospitalized for COVID-19?

PICO 3: What is the risk of severe COVID-19 disease and death amongst patients with chronic kidney disease?

\section{Search strategy}

The methods team searched the following electronic databases: Embase, PubMed, Epistemonikos, and Cochrane from January 1st, 2020 to January 5th, 2021. Additionally, the investigators searched MEDRXIV, SSRN, and LiTCOVID databases for preprints of unpublished reviews. The detailed search strategy is available in Appendix-1 (See Online Supplementary material). In addition, reviewers manually checked the reference lists of included studies to identify additional relevant publications. The investigators further extended the search to include primary studies that were not incorporated in the systematic reviews from September 1st, 2020 to January 10th, 2021. The investigators also included results from four registries in this review: Hilbrands 2020 [13], Holman 2020 [14], Jager 2020 [15], and Williamson 2020 [16]. Similarly, reviewers assessed the references of included primary studies to identify additional publications that were not captured in the original search.

\section{Data collection}

Four investigators (AA, RM, AG, SJ) independently performed title and abstract screening in pairs to identify eligible literature. When present, disagreements were resolved by a third investigator (RAM). After full-text screening, four reviewers extracted data from the included systematic reviews independently (AA, RM, AG, SJ).

We collected the following information from each review: study characteristics (author name, region/country, study design, inclusion and exclusion criteria), patient characteristics (number of patients with CKD, age, gender, comorbidities, and clinical setting), and CKD specifications (CKD stage and whether they included patients with end stage kidney disease (ESKD) or not). We extracted the adjusted effect estimates when available with $95 \%$ confidence interval (CI) including odds ratio (OR), relative risk (RR), and hazard ratio (HR) for the following outcomes: incidence of COVID-19 infection, hospitalization, severe illness, ICU admission, mechanical ventilation, mortality, and poor outcomes among patients with CKD and COVID-19 infection from both systematic reviews and primary studies.

\section{Quality and risk of bias assessment}

We evaluated the quality of included systematic reviews using the modified Assessment of the methodological quality of systematic reviews (AMSTAR) tool checklist [17], and applying the following criteria: availability of a study protocol, comprehensive search strategy, list of excluded studies and their reason for exclusion, risk of bias $(\mathrm{RoB})$ assessment and evaluation of its impact, appropriate methods for statistical combination of results, and assessment of publication bias.

When more than one review addressed the same question, we prioritized reviews that fulfilled most of the following criteria: higher AMSTAR rating, peer reviewed, recent date of literature search, use of the Grading of Recommendations, Assessment, Development and Evaluations (GRADE) assessment. We also prioritized reviews that addressed the outcomes of interest in the most direct way. Appendix 2A (See Online Supplementary material) provides details about the AMSTAR evaluation of all included systematic reviews. 
Fig. 1 Flow Chart of a systematic reviews and $\mathbf{b}$ primary studies included in the review

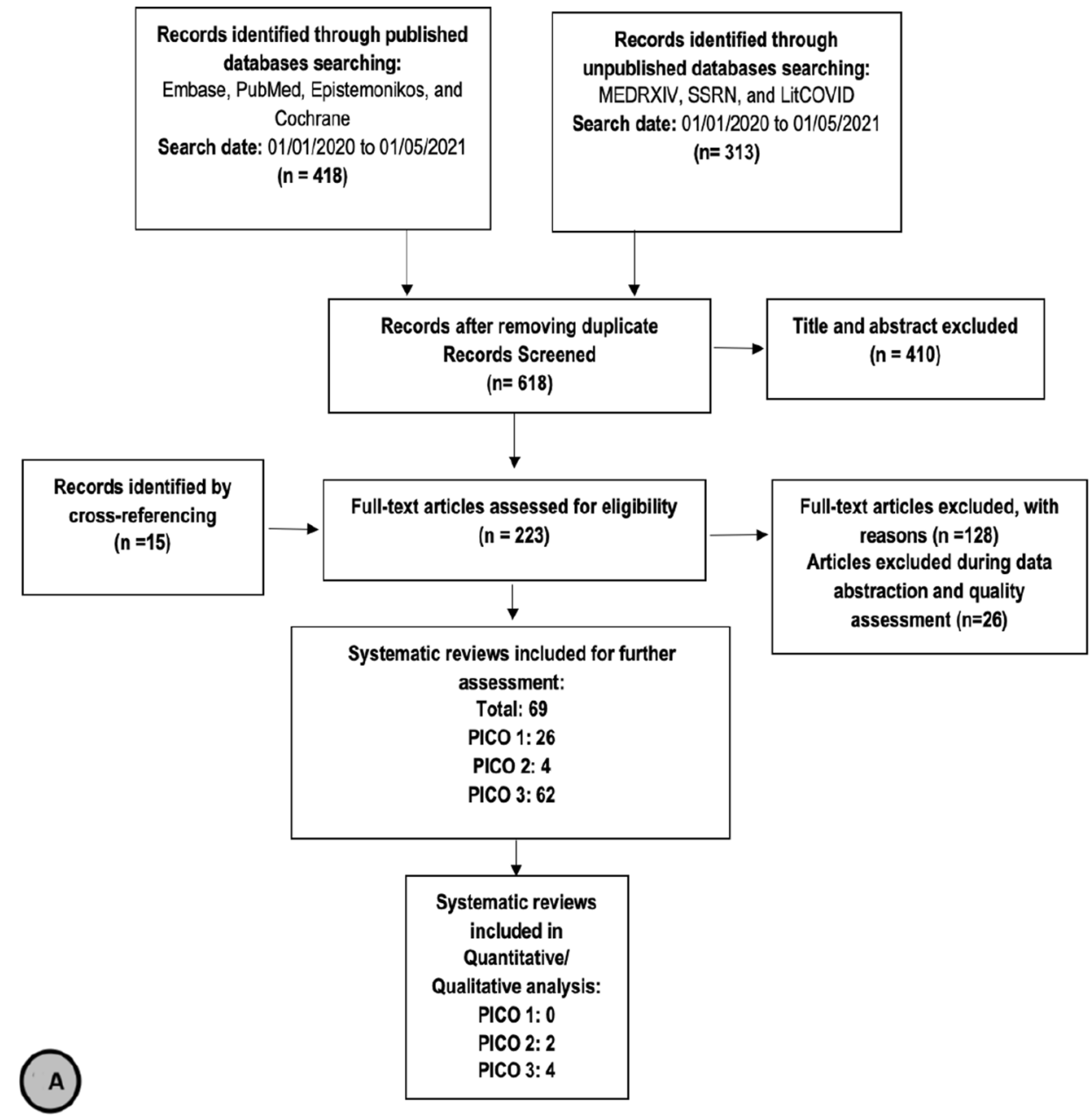

We evaluated the risk of bias of the primary studies using the Quality in Prognostic Studies (QUIPS) tool [18]. The QUIPS tool covers six domains: selection bias, attrition bias, prognostic factor and outcome measurement, confounding, and bias related to statistical analysis or presentation of results. The quality of each study was categorized as low risk, moderate risk, and high risk for each of the six domains. Appendix 2B (See Online Supplementary material) provides details about the QUIPS RoB evaluation for the included primary studies.

\section{Certainty of the evidence}

We assessed the certainty in the evidence using the GRADE approach [19]. This approach has four levels of certainty; very low, low, moderate and high. Observational studies start at low certainty and can be downgraded for concerns of risk of bias, indirectness (applicability of the results to the question), inconsistency (heterogeneity between study results), imprecision, and publication bias, while it can be upgraded if there is a large effect, residual confounding effect, or dose-response gradient.

\section{Statistical analysis}

Quantitative and descriptive analyses were conducted. We summarized the characteristics of the included systematic reviews and primary studies. Moreover, overall ORs, HRs, and RRs, along with their respective 95\% CIs of the mortality outcome in patients diagnosed with CKD versus without CKD diagnosis and COVID-19 were calculated from the additional primary studies and the studies included in the reviews using a random effect model when more than five studies were available. Study data was considered worthy of exploration of heterogeneity when the $\mathrm{I}^{2}$ statistic was more than 50\%. Attempts were also made to explain heterogeneity based on the patients' clinical characteristics. We explored potential publication bias for the mortality outcome in studies through funnel plots. Reviewers eyeballed the plots to assess their symmetry. Subgroup analyses were conducted according to CKD classification status (stage 3, 4, or 5). All analyses were performed using Review Manager (RevMan) software version 5.4. 
Fig. 1 (continued)

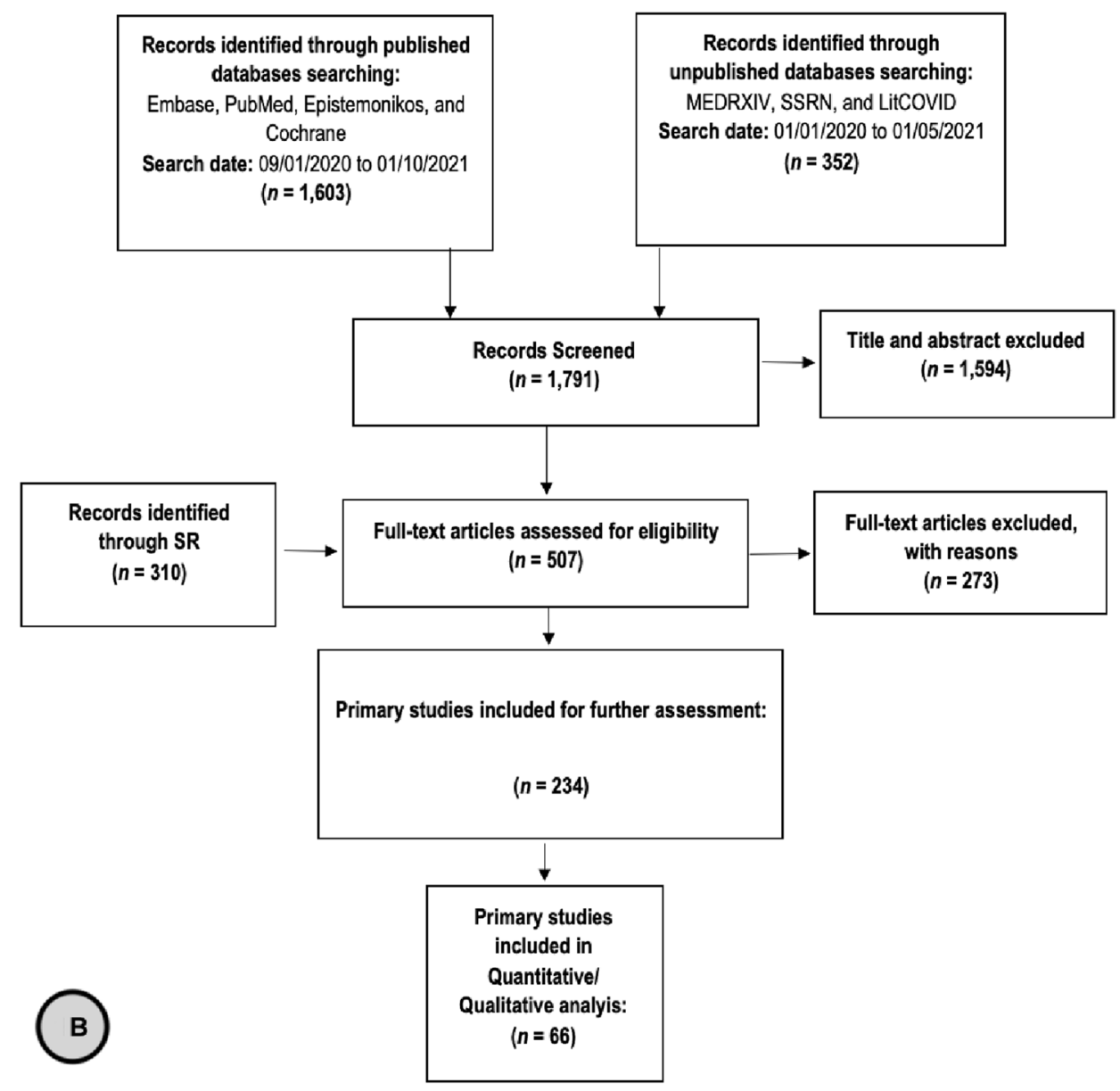

\section{Results}

We Identified 731 records through published and unpublished databases. After removing duplicates, we screened a total of 618 reviews for title and abstract screening. Two hundred and twenty-three reviews were included for full text screening, during which a total of 69 systematic reviews were included for prioritization: twenty-six reviews reported indirect evidence that informed $\mathrm{PICO}-1$, four reviews addressed the risk of hospitalization in patients with $\mathrm{CKD}$ and COVID-19 (PICO-2), and 62 reviews addressed the risk of mortality in patients with CKD and COVID-19 (PICO-3). After prioritization of the 69 reviews, we included six reviews in our final report (Figure 1A). We also identified and screened 1,791 primary studies for eligibility, of which 234 studies were used for further assessment and meta-analysis. We included 66 primary studies in our final report. Table 1 summarizes the effect estimates for the different outcomes in the included systematic reviews and primary studies. The characteristics as well as outcome measurements reported in the systematic reviews and primary studies are detailed in appendix 3 (Tables 3 A-D) (See Online Supplementary material).

\section{CKD and contracting COVID-19}

We did not identify any systematic reviews that directly inform on CKD and the risk of contracting Covid-19. We identified 28 systematic reviews [2, 20-46] that reported on the prevalence of CKD among patients with COVID-19, which ranged from 0.4 to $49.0 \%$ among different settings. Two [27, 32] reviews reported on the prevalence of ESKD in patients with COVID-19 infection which ranged from 2.3 to $30.9 \%$. One review [33] reported an $8 \%$ incidence of COVID-19 infection in patients on chronic hemodialysis (95\% CI 4.7-12.0\%).

With regard to additional primary studies that were not included in the reviews, there was no convincing difference on the risk of acquiring COVID-19 infection in patients with and without CKD, with inconsistent results being present among different studies. While Rentsch et al. showed no difference in the OR for testing positive for COVID-19 infection in patients with and without CKD, OR $1.00(95 \%$ CI 0.76-1.33) [47], Ji et al. reported an OR of 0.50 (95\% CI 0.39-0.65) [48] when examining the relationship between CKD and the presence of COVID-19, and Corbett et al. [49] reported on the rate of COVID-19 infection over a six-week 


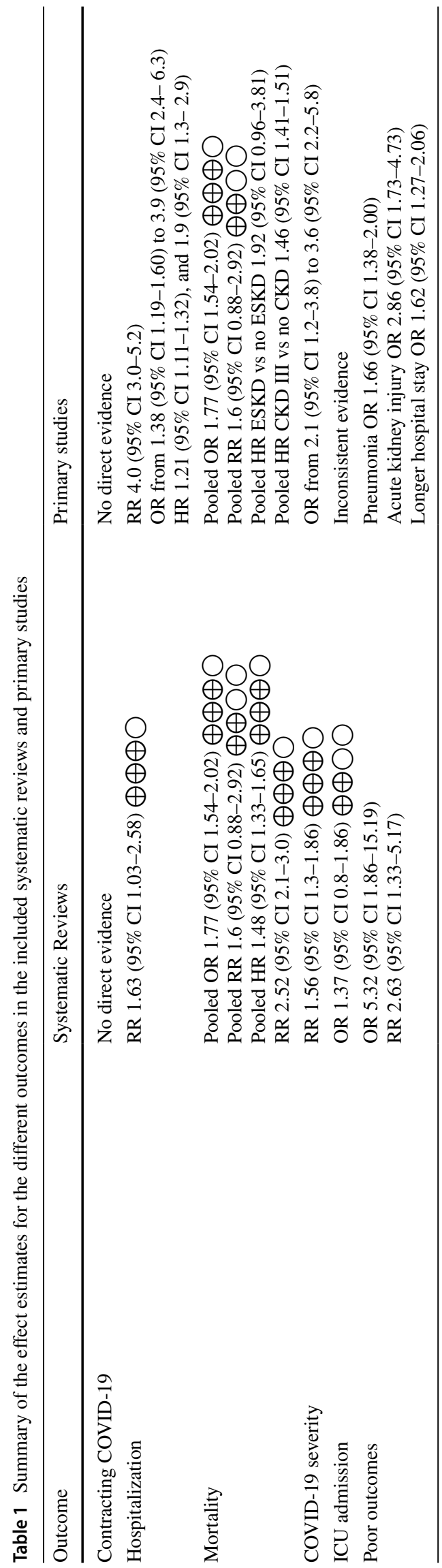

period in a large urban dialysis center in the United Kingdom with 1530 patients. During this period, $19.6 \%$ of the dialysis patients developed COVID-19 infection, with the majority of cases (96\%) being in patients on in-center dialysis compared to home dialysis patients.

\section{CKD and the risk of hospitalization among patients with COVID-19}

We prioritized two of the four systematic reviews addressing this question [50, 51]. Fernandez Villalobos [50] provided the needed information to assess certainty in evidence. The risk of hospitalization appears to be increased in patients with COVID-19 infection and CKD compared to those without $\mathrm{CKD}, \mathrm{RR}=1.63$ (95\% CI 1.03-2.58) (Moderate certainty) [50] (Table 2).

Concerning additional primary studies that were not included in the reviews, most of the studies that reported on hospitalization in patients with COVID-19 infection and CKD showed a statistically significant increase in the risk of hospitalization. The majority of these studies calculated the OR for hospitalization, which ranged from 1.38 (95\% CI $1.19-1.60)$ to 3.9 (95\% CI 2.4-6.3) [47, 52-58]. One primary study reported a RR of 4.0 (95\% CI 3.0-5.2) for hospitalization in patients with COVID-19 infection and CKD [59]. Two studies reported a HR of 1.21 (95\% CI 1.11-1.32), and 1.9 (95\% CI 1.3-2.9) [60, 61].

Oetjens et al. analyzed the risk of hospitalization stratified by the advancement of CKD, showing an incremental increase in the odds of hospitalization in patients with COVID-19 infection with advancing CKD stage [54]. The OR for hospitalization in patients with CKD stage 5 and on dialysis was 11.07 (95\% CI 4.54-26.97) in the Geisinger health care system, and 8.83 (95\% CI 2.76-28.27) in United States Renal Data System (USRDS) data [54].

\section{CKD and mortality among patients with COVID-19}

We identified 62 systematic reviews assessing mortality risk in patients with CKD who contracted COVID-19 [2, 20, $24,40,43,44,46,50,51,62-88$ ]. Dorjee et al. [46] was the most updated systematic review with good AMSTAR quality which showed higher RR of mortality in patients with CKD and COVID-19 infection, $\mathrm{RR}=2.52$, (95\% CI 2.11-3.00) (Moderate certainty) (Table 2).

We identified 20 primary studies assessing the HR of mortality in patients with CKD and COVID-19 [14, 16, 57, 60, 90-104], with a pooled HR of 1.48 (95\% CI 1.33-1.65) (Moderate certainty) compared to patients without CKD (Fig. 2A). Holman et al. showed that there is an increase in the HR of mortality with advanced CKD among patients with type 1 diabetes, with a HR of 2.07 (95\% CI 1.48-2.89), 2.46 (95\% CI 1.72-3.52), 3.71 (95\% CI 2.47-5.58), and 


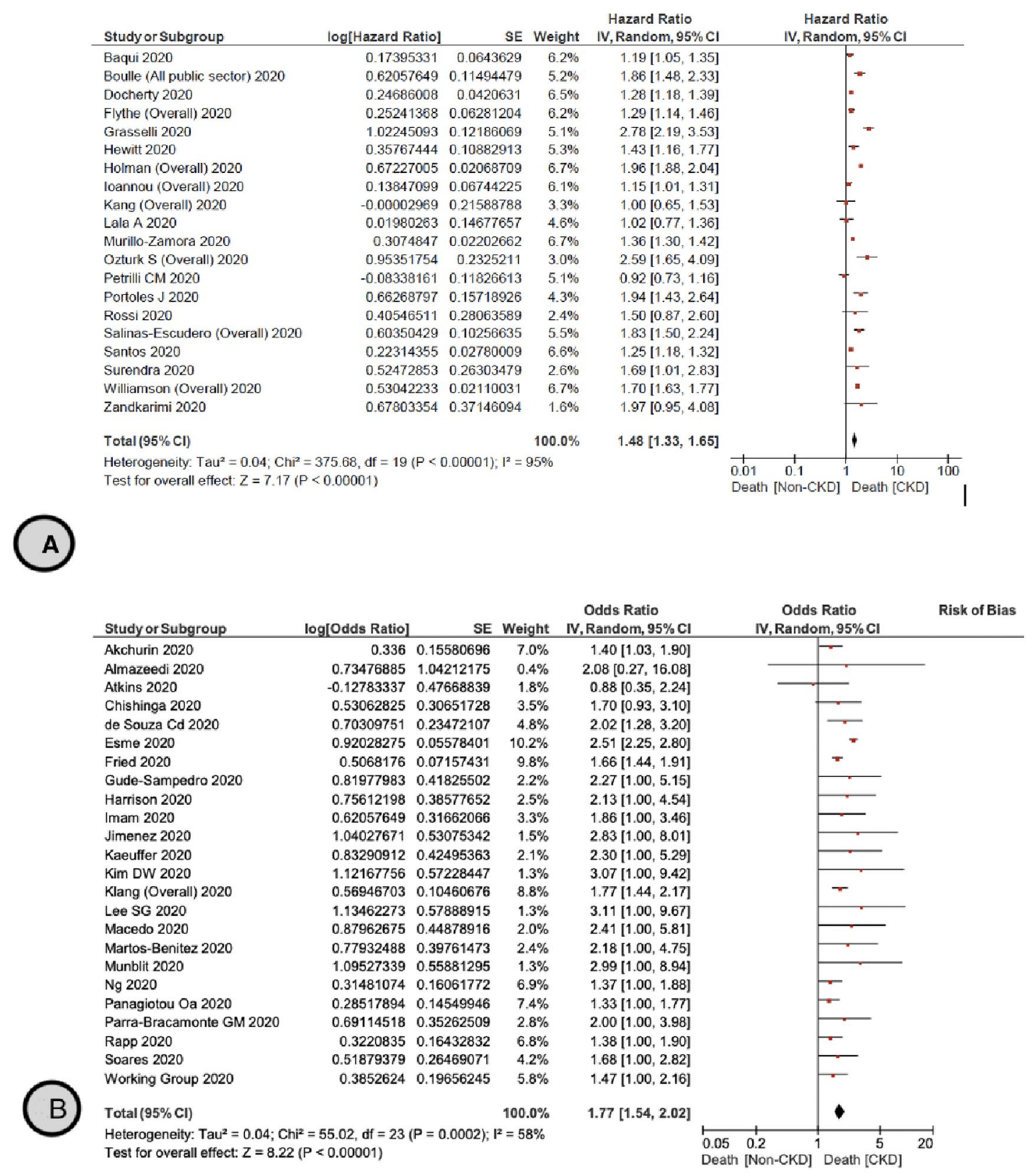

Fig. 2 A The pooled hazard ratio for mortality for patients with and without CKD. B The pooled odds ratio for mortality for patients with and without CKD. C The pooled risk ratio for mortality for patients

8.35 (95\% CI 5.50-12.70) in CKD stages 3A, 3B, 4, and 5, respectively [14]. Patients with type 2 diabetes had a similar increase in mortality risk with advanced CKD, with a HR of 1.39 (95\% CI 1.30-1.49), 1.76 (95\% CI 1.63-1.89), 2.31 (95\% CI 2.10-2.54), and 4.91 (95\% CI 4.34-5.56) in CKD stages $3 \mathrm{~A}, 3 \mathrm{~B}, 4$, and 5, respectively [14]. This incremental increase in mortality was consistent with findings in Williamson et al. which showed higher HR 3.69 (95\% CI 3.09-4.39) for mortality in patients with ESKD compared to those without ESKD [16]. with and without CKD. D The pooled Hazard ratio for mortality for patients with CKD stage 3, 4-5 and without CKD. E The pooled Hazard ratio for mortality for patients with and without ESKD

We also identified 24 additional primary studies that were not included in the reviews [52, 53, 55, 56, 58, 106-123] that examined the OR of mortality in patients with CKD and COVID-19 infection with a pooled OR of 1.77 (95\% CI 1.54-2.02) (Moderate certainty) compared to patients without CKD (Fig. 2B). Ng et al. [123] reported specifically on patients with ESKD, showing a higher RR of mortality in this population, OR 1.37 (95\% CI 1.09-1.73). Additionally, three primary studies [125-126] reported on the RR of mortality in patients with CKD and COVID-19 infection 


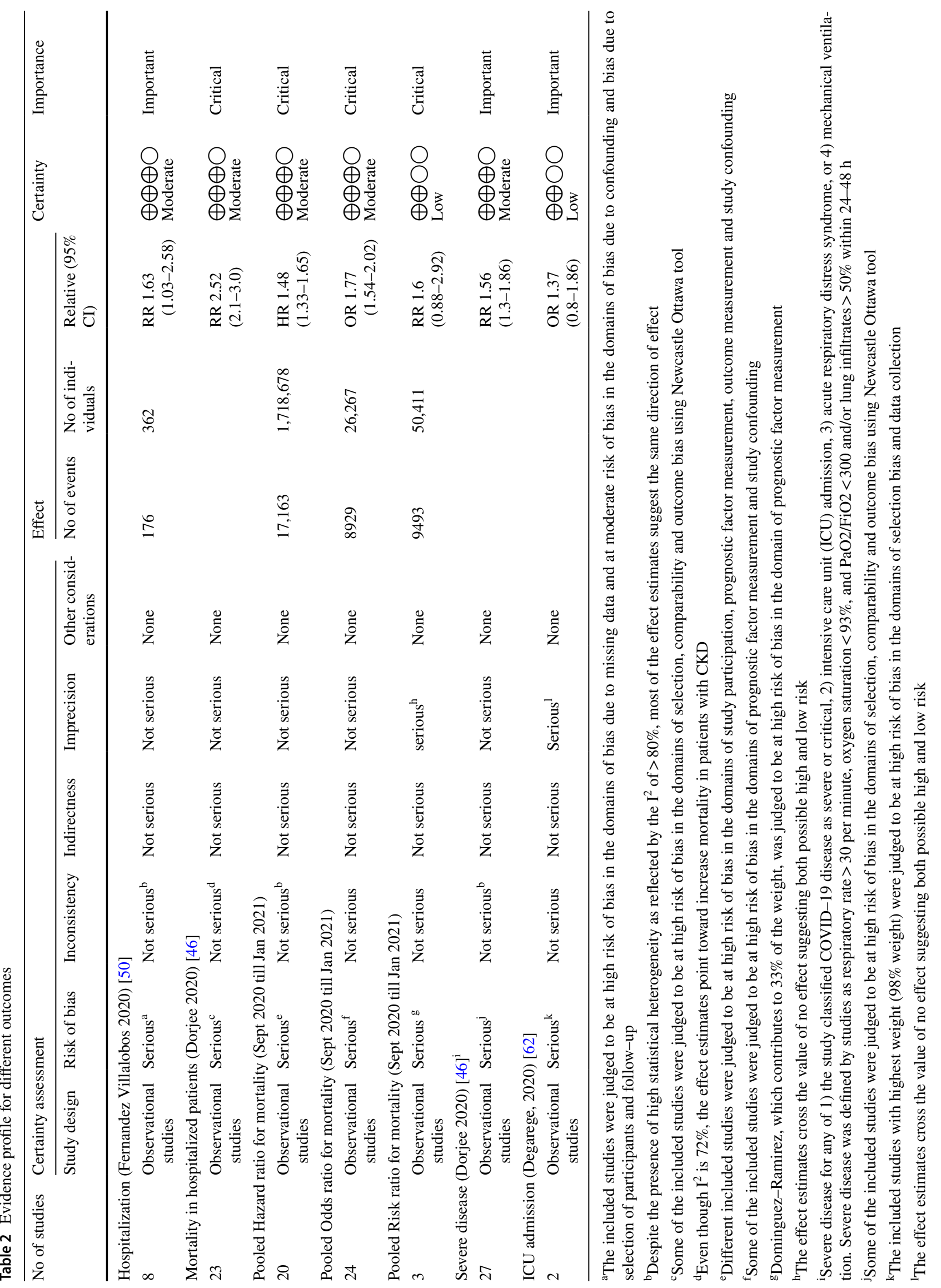




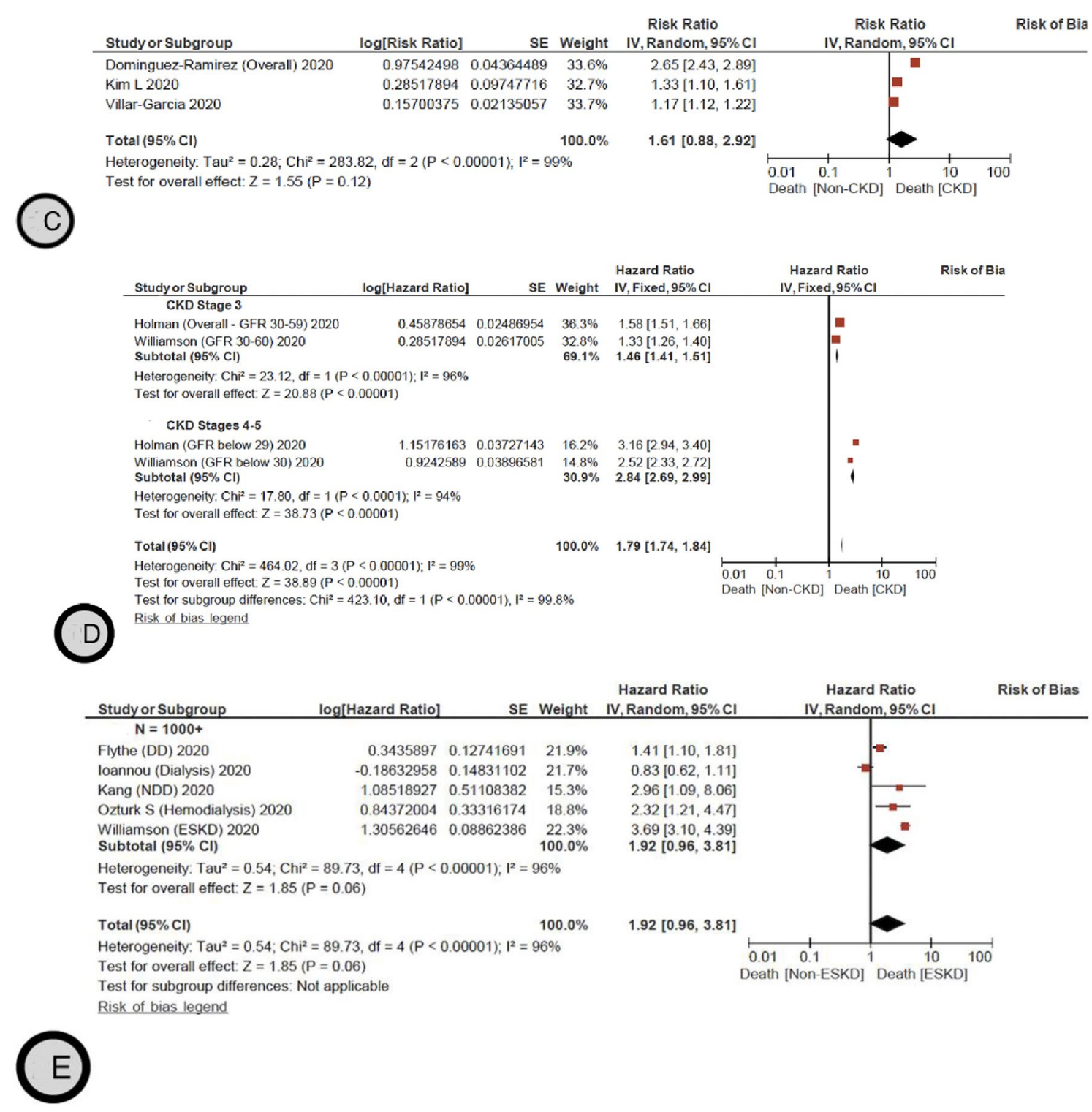

Fig. 2 (continued)
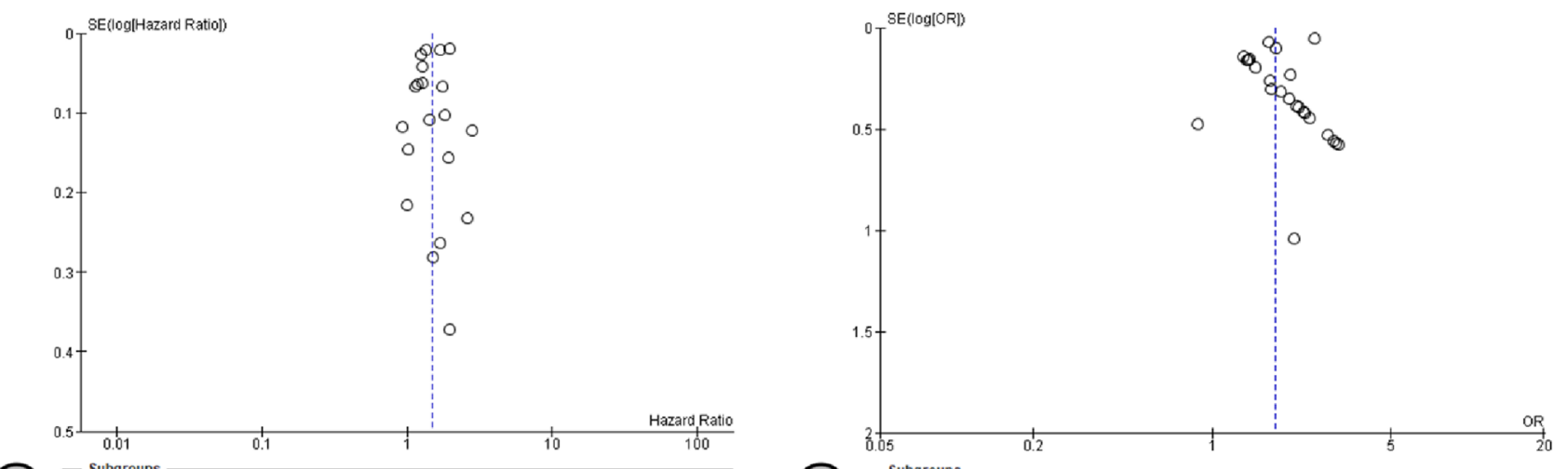

$\mathrm{O}_{\mathrm{N}=1000+}^{\text {Subgroups }}$

$\Gamma^{S} \mathrm{~N}=1000+$

Fig. 3 A Funnel plot of comparison: 1 Covid-19 and CKD Mortality Outcomes, outcome: 1.1 HR CKD vs Non-CKD Mortality. B Funnel plot of comparison: 1 Covid-19 and CKD Mortality Outcomes, outcome: 1.2 OR CKD vs Non-CKD Mortality 
with a pooled RR of 1.6 (95\% CI 0.88-2.92) (Low certainty) (Fig. 2C). Jager et al. [15] reported that the attributable mortality was $20 \%$ and the mortality risk was 21.1 (95\% CI 18.6-23.9) times higher in dialysis patients diagnosed with COVID-19 compared with the $1.2 \%$ mortality in the matched control group of dialysis patients without COVID19. There was no suspected publication bias in the studies reporting mortality (Fig. 3).

In a subgroup analysis, the pooled HR for mortality in patients with CKD stage 3 is 1.46 (95\% CI 1.41-1.51) compared to patients without CKD [14, 16] (Fig. 2D). Interestingly, the pooled HR for mortality significantly increased in patients with CKD 4-5, HR 2.84 (95\% CI 2.69-2.99) [14, 16] (Fig. 2D). This increased mortality risk was also persistent in patients with ESKD with a pooled HR of 1.92 (95\% CI 0.96-3.81) compared to patients without ESKD [16, 60, 93, 101, 103] (Fig. 2E).

\section{CKD and COVID-19 severity}

We identified 32 systematic reviews $[2,20,23,24,28$, $30,35,36,43,46,50,51,62,82,128-144]$ reporting on COVID-19 severity in patients with CKD. Dorjee et al. [46] was the most updated systematic review with good AMSTAR quality answering this question and it showed an increased risk of severe COVID-19 infection among patients with CKD with a RR of 1.6 (95\% CI 1.3-1.9) (Moderate certainty) (Table 2).

Five additional primary studies that were not included in the reviews $[48,52,107,145,146]$ examined the effect of CKD on the severity of COVID-19 infection, showing a higher OR for severe COVID-19 infection ranging from 2.1 (95\% CI 1.2-3.8) to 3.6 (95\% CI 2.2-5.8). However, the definition of severe COVID-19 infection was not consistent among different studies.

\section{CKD and ICU admission among patients with COVID-19}

We identified nine systematic reviews $[2,29,40,50,62$, $79,137,142,143]$ reporting on ICU admission in patients with CKD and COVID-19 infection. Among them, Degarege A [62] was the most updated systematic review with the highest AMSTAR quality. Degarege reported a trend towards higher need for ICU admission in patients with CKD and COVID-19 infection with an OR of 1.37 (95\% CI 0.88-1.86) (Low certainty) (Table 2).

The effect estimate for the need of ICU admission was inconsistent among additional primary studies that were not included in the reviews. While three studies [52, 108, 147] showed a statistically significant increase in the risk of ICU admission with an OR ranging from 1.7 to 3.6, the other five studies $[47,53,55,119,126]$ did not provide convincing evidence for higher ICU admission need in patients with CKD and COVID-19 infection (Table 1).

\section{CKD and mechanical ventilation among patients with COVID-19}

We identified one relevant systematic review [79] that reported on the risk of mechanical ventilation need in patients with CKD and COVID-19 infection. The meta-analysis included one primary study which did not show convincing evidence that $C K D$ increases the need for mechanical ventilation in this population based on 18 events only.

There was also no convincing evidence that CKD increases the risk of needing mechanical ventilation among patients with COVID-19 infection in five additional primary studies that were not included in the reviews $[53,60,93$, $123,148]$. The ORs for mechanical ventilation ranged from 0.97 to 1.32 , and the HRs ranged from 0.87 to 1.16 .

\section{CKD and poor outcomes among patients with COVID-19}

We identified four systematic reviews [22, 79, 83, 149] reporting on overall poor outcomes in patients with CKD and COVID-19 infection. Nandy, K. et al. [22] defined adverse events such as ICU admission, acute respiratory distress syndrome (ARDS), mechanical ventilation, pneumonia, and death. The OR for adverse events was higher in CKD patients compared to non-CKD OR 5.32 (95\% CI 1.86-15.19). Pranata R [79] defined poor outcomes as mortality, severe COVID-19, ARDS, ICU care, and mechanical ventilation, and showed a RR of 2.63 (95\% CI 1.33-5.17) for the CKD population compared to non-CKD. Xiao, W et al. defined adverse outcomes as severe illness, critical illness or death and reported a pooled effect estimate of 1.64 (95\% CI 1.28-2.09) [83]. Kunutsor et al. also reported a higher incidence of AKI in patients with baseline CKD [27].

Additional primary studies that were not included in the reviews showed that patients with CKD and COVID-19 infection are at higher risk of pneumonia, OR 1.66 (95\% CI 1.38-2.00) [53] and acute kidney injury in the first $48 \mathrm{~h}, \mathrm{OR}$ 2.86 (95\% CI 1.73-4.73) [150]. One study reported longer hospital stay in patients with ESKD compared to non-dialysis CKD, OR 1.62 (95\% CI 1.27-2.06) [123].

\section{Discussion}

COVID-19 has affected millions of people worldwide. Many chronic medical diseases were reported as risk factors for increased mortality and severity of COVID-19, such as diabetes [56], hypertension, chronic obstructive 
pulmonary disease, malignancies, and CKD [127]. In 2019, CKD affected approximately $15.0 \%$ of patients aged 65 years or older of the US Medicare population [151]. Some of the major causes of morbidity and mortality in patients with CKD are infections, sepsis, and bacteremia [152]. Infections in patients with CKD can cause longer duration of hospitalization, [153] and the mortality rate from pneumonia in patients with CKD is higher than that of patients without CKD [154]. In our review, we gathered evidence from all systematic reviews and primary studies to report the impact of CKD on COVID-19 mortality, hospitalization, incidence, ICU admission, disease severity, and adverse outcomes. We found that patients with CKD were more likely to have worse outcomes from COVID-19 compared to patients without CKD. This could be attributed to the attenuated immune system activation of both the innate and adaptive immunity systems which lead to an increased susceptibility to infections in patients with CKD [155].

We are reporting moderate certainty evidence that CKD increases the risk of COVID-19-related mortality and of disease severity. Importantly, this mortality risk is higher in patients with advanced CKD stage (Figs. 2 D, E).

In comparison with the impact of other comorbidities on COVID-19 mortality, a meta-analysis showed that cardiovascular disease, hypertension and diabetes were associated with increased mortality and severity of COVID-19: diabetes OR 2.50 (95\% CI 1.74-3.59), and OR 2.35 (95\% CI 1.80-3.06), hypertension OR 2.88 (95\% CI 2.22-3.74), and OR 2.98 (95\% CI 2.37-3.75), and cardiovascular disease OR 6.34 (95\% CI 3.71-10.84), and OR 4.02 (95\% CI 2.76-5.86), respectively for mortality and severity [156]. Another meta-analysis reported that patients with diabetes mellitus RR 1.48 (95\% CI 1.02-2.15), cardiovascular diseases RR 2.25 (95\% CI 1.60-3.17), malignancy RR 1.47 (95\% CI 1.01-2.14), and hypertension RR 1.82 (95\% CI 1.43-2.32) suffer a greater mortality risk compared to patients without these comorbidities [81].

Our findings show moderate certainty evidence that risk of hospitalization is increased in patients with COVID-19 infection and CKD compared to those without CKD. Similar to mortality, there is an incremental increase in the risk of hospitalization with advanced CKD stage [54]. In a casecontrol study that assessed risk factors associated with increased hospitalization in patients with CKD, the presence of comorbid ischemic heart disease was associated with a 3.5 fold increase in admission rate (95\% CI 2.14-5.9), while other factors included the presence of anemia, hypoalbuminemia, and late referral to a nephrologist [157].

The significance of CKD as an underlying condition for severe COVID-19 remains less well understood. In our review, we found one high quality systematic review [46] and five primary studies $[48,52,107,145,146]$ that reported an increased risk of severe COVID-19 disease in patients with CKD, with an OR ranging from 2.1 (95\% CI 1.2-3.8) to 3.6 (95\% CI 2.2-5.8). However, the definition of severe COVID-19 infection was not clear and likely inconsistent in all studies. In one meta-analysis, no primary study reported CKD as a risk factor for COVID-19 severity, but a significant association was found, OR 3.03 (95\% CI, 1.09-8.47) when pooling of data took place [128].

It is worthy to note that the results informing some of the outcomes were inconsistent among studies. For example, the effect estimates for the need of ICU admission and poor outcomes in patients with CKD and COVID-19 were inconsistent among the identified primary studies. Moreover, some inconsistencies in the inclusion of primary studies among the published reviews were noted. These discrepancies can be attributed to several possible factors, like the use of different definitions of CKD and disease severity, the use of different inclusion criteria in the systematic reviews, the difference in sample sizes and the timing of the studies, and different management and care that is provided to patients in each study. In addition, some studies did not adjust for all appropriate confounders, which may have played a role in the inconsistencies among results.

The findings in this review and in other studies have shed some light on the importance of implementing clear guidelines for the prevention and management of COVID-19 that are specific to patients with CKD. Because studies on these patients have shown an increased risk of mortality, hospitalization, and adverse outcomes of COVID-19, physicians should maintain a low threshold for hospital admission and close monitoring of patients with CKD who are not hospitalized, as well as early aggressive management to prevent complications. The findings should also guide us to prioritize patients with CKD during vaccine administration, regardless of their age and the advancement of their disease. Recent literature on vaccinated patients on maintenance hemodialysis showed that those patients have mounted an immune response to the vaccine, however, their antibody titers were lower than their controls [158, 159]. Studies examining the protective effect of the COVID-19 vaccine in CKD patients are underway.

\section{Limitations}

Some limitations to our overview of reviews could be noted. First, we relied on existing systematic reviews to identify studies published before September 2020. Given the inconsistency in studies included among the published reviews that is unexplained by the reviews' inclusion and exclusion criteria, it is possible that some primary studies may have been missed. However, due to the extensive effort in identifying large and well-done studies, it is unlikely that 
any major study that would have a considerable impact on the conclusions has been missed. Some of the systematic reviews and primary studies were preprints, which lack the vigilant peer-review process. Another limitation is the high risk of bias in multiple domains in the included primary studies, and some primary studies did not consistently adjust for important confounders. In addition, the methods of diagnosing chronic kidney disease and measuring different confounders were not explicitly detailed in most of the included primary studies. In the mortality outcome, study data were considered worthy of exploration of heterogeneity when the $\mathrm{I}^{2}$ statistic was more than $50 \%$. Attempts were also made to explain heterogeneity based on the patients' clinical characteristics. However, due to lack of reporting of factors that may explain heterogeneity in the included studies, we were unable to explore them for all the outcomes in our analysis.

\section{Conclusions}

This overview of reviews addressed systematic reviews and primary studies that evaluated different outcomes in patients with CKD who contracted COVID-19. Our overview also evaluated the quality of both systematic reviews and individual studies. Evidence consistently demonstrated an increased risk of mortality and hospitalization in patients with CKD and COVID-19. The extent to which CKD increases the likelihood of the rate of infection, and other poor outcomes is not currently well understood, and the results are inconsistent among studies. The results shed some light on the significance of prioritizing patients with CKD for COVID-19 vaccination and critical care management. Further research studying the pathophysiology behind the effect of CKD on COVID-19 outcomes would provide deeper insight for the management of such patients.

Supplementary Information The online version contains supplementary material available at https://doi.org/10.1007/s40620-021-01206-8.

Author contributions Conception or design of the work: RM. Data collection and interpretation: All Authors, Data analysis: PT. Drafting the article: SJ, RM, AE, RM. Critical revision of the article: All Authors. Final approval of the version to be published: All authors.

Funding This work informed a brief for the World Health organization and was funded through the Pan American Health Organization (PAHO).

Availability of data and material All data generated or analyzed during this study are included in this published article and its supplementary information files.

Code availability Not applicable.

\section{Declarations}

Conflict of interest All the authors declared no conflict of interest/ competing interests.

Ethical approval Not applicable.

Consent to participate Not applicable.

Consent for publication Not applicable.

\section{References}

1. https://covid19.who.int/. WHO website. In:2021.

2. Zhou Y, Yang Q, Chi J et al (2020) Comorbidities and the risk of severe or fatal outcomes associated with coronavirus disease 2019: a systematic review and meta-analysis. Int J Infect Dis 99:47-56

3. Zaki N, Mohamed EA, Ibrahim S, Khan G (2020) The influence of comorbidity on the severity of COVID-19 disease: systematic review and analysis. medRxiv. 2020:20134478

4. GBDCKD Collaboration (2020) Global, regional, and national burden of chronic kidney disease, 1990-2017: a systematic analysis for the Global Burden of Disease Study 2017. Lancet 395(10225):709-733

5. Centers for Disease Control and Prevention (2021) Chronic Kidney Disease in the United States. Atlanta GUDoHaHS, Centers for Disease Control and Prevention; 2021. Chronic Kidney Disease in the United States, 2021. US Department of Health and Human Services, Centers for Disease Control and Prevention, Atlanta

6. https://kdigo.org/wp-content/uploads/2016/10/KDIGO-2012AKI-Guideline-English.pdf. KDIGO guidelines. 2021.

7. Go AS, Chertow GM, Fan D, McCulloch CE, Hsu C-Y (2004) Chronic kidney disease and the risks of death, cardiovascular events, and hospitalization. New Engl J Med 351(13):1296-1305

8. Dalrymple LS, Go AS (2008) Epidemiology of acute infections among patients with chronic kidney disease. Clin J Am Soc Nephrol 3(5):1487-1493

9. de Jager DJ, Grootendorst DC, Jager KJ et al (2009) Cardiovascular and noncardiovascular mortality among patients starting dialysis. JAMA 302(16):1782-1789

10. Fried LF, Katz R, Sarnak MJ et al (2005) Kidney function as a predictor of noncardiovascular mortality. J Am Soc Nephrol 16(12):3728-3735

11. Chang C-H, Fan P-C, Kuo G et al (2020) Infection in advanced chronic kidney disease and subsequent adverse outcomes after dialysis initiation: a nationwide cohort study. Sci Rep 10(1):2938

12. Liberati A, Altman DG, Tetzlaff J et al (2009) The PRISMA statement for reporting systematic reviews and meta-analyses of studies that evaluate healthcare interventions: explanation and elaboration. BMJ 339:b2700

13. Hilbrands LB, Duivenvoorden R, Vart P et al (2020) COVID19-related mortality in kidney transplant and dialysis patients: results of the ERACODA collaboration. Nephrol Dial Transplant 35(11):1973-1983

14. Holman N, Knighton P, Kar P et al (2020) Risk factors for COVID-19-related mortality in people with type 1 and type 2 diabetes in England: a population-based cohort study. Lancet Diabetes Endocrinol 8(10):823-833 
15. Jager Kj KACNCCCS-AJEGLCFHMHAPKJL (2020) Results from the ERA-EDTA Registry indicate a high mortality due to COVID-19 in dialysis patients and kidney transplant recipients across Europe. Kidney Int. https://doi.org/10.1016/j.kint.2020. 09.006

16. Williamson EJ, Walker AJ, Bhaskaran K et al (2020) Factors associated with COVID-19-related death using OpenSAFELY. Nature 584(7821):430-436

17. Shea BJ, Reeves BC, Wells G et al (2017) AMSTAR 2: a critical appraisal tool for systematic reviews that include randomised or non-randomised studies of healthcare interventions, or both. BMJ 358:j4008

18. Hayden JA, van der Windt DA, Cartwright JL, Côté P, Bombardier C (2013) Assessing bias in studies of prognostic factors. Ann Intern Med 158(4):280-286

19. Guyatt GH, Oxman AD, Vist GE et al (2008) GRADE: an emerging consensus on rating quality of evidence and strength of recommendations. BMJ 336(7650):924-926

20. $\mathrm{Ma} \mathrm{C}, \mathrm{Gu} J$, Hou P et al (2020) Incidence, clinical characteristics and prognostic factor of patients with COVID-19: a systematic review and meta-analysis. medRxiv 41:145

21. Bennett S, Tafuro J, Mayer J et al (2021) Clinical features and outcomes of adults with coronavirus disease 2019: a systematic review and pooled analysis of the literature. Int J Clin Pract 2020:e13725

22. Nandy K, Salunke A, Pathak SK et al (2020) Coronavirus disease (COVID-19): A systematic review and meta-analysis to evaluate the impact of various comorbidities on serious events. Diabetes Metab Syndr 14(5):1017-1025

23. Oyelade T, Alqahtani J, Canciani G (2020) Prognosis of COVID19 in patients with liver and kidney diseases: an early systematic review and meta-analysis. Trop Med Infect Dis. https://doi.org/ 10.3390/tropicalmed5020080

24. Singh AK, Gillies CL, Singh R et al (2020) Prevalence of comorbidities and their association with mortality in patients with COVID-19: a systematic review and meta-analysis. Diabetes Obes Metab 22(10):1915-1924

25. Khan MMA, Khan MN, Mustagir MG, Rana J, Islam MS, Kabir MI (2020) Effects of underlying morbidities on the occurrence of deaths in COVID-19 patients: a systematic review and metaanalysis. J Glob Health 10(2):020503

26. Fang M, Wang D, Tang O, Selvin E (2020) Prevalence of chronic disease in laboratory-confirmed covid-19 cases and US adults (2017-2018). Diabetes Care 43(10):e127

27. Kunutsor SK, Laukkanen JA (2020) Renal complications in COVID-19: a systematic review and meta-analysis. Ann Med 52(7):345-353

28. Fathi M, Vakili K, Sayehmiri F et al (2020) Prognostic value of comormidity for severity of covid-19: a systematic review and meta-analysis study. medRxiv 12:6049

29. Chang R, Elhusseiny KM, Yeh Y-C, Sun W-Z (2020) COVID19 ICU and mechanical ventilation patient characteristics and outcomes - a systematic review and meta-analysis. medRxiv 16(2):e0246318

30. Meng Y, Wang J, Wen K, Da W, Yang K, Zhou S, Tao Z, Liu H, Tao L (2021) Clinical Features and Laboratory Examination to Identify Severe Patients with COVID-19: A Systematic Review and Meta-Analysis. BioMed Res Int 2021:6671291

31. Bajwa H, Riaz Y, Ammar M, Farooq S, Yousaf A (2020) The dilemma of renal involvement in Covid-19: a systematic review. Cureus. 12(6):e8632

32. Zhou S, Xu J, Xue C, Yang B, Mao Z, Ong ACM (2020) Coronavirus-associated kidney outcomes in COVID-19, SARS, and MERS: a meta-analysis and systematic review. Ren Fail 43(1):1-15
33. Tan E, Song J, Deane AM, Plummer MP (2021) Global impact of coronavirus disease 2019 infection requiring admission to the icu: a systematic review and meta-analysis. Chest 159(2):524-536

34. Bajgain KT, Badal S, Bajgain BB, Santana MJ (2021) Prevalence of comorbidities among individuals with COVID-19: a rapid review of current literature. Am J Infect Control 49(2):238-246

35. Zhou Y, Ren Q, Chen G et al (2020) Chronic kidney diseases and acute kidney injury in patients with COVID-19: evidence from a meta-analysis. Front Med (Lausanne) 7:588301-588301

36. Wu T, Zuo Z, Kang S et al (2020) Multi-organ dysfunction in patients with COVID-19: a systematic review and meta-analysis. Aging Dis 11(4):874-894

37. Emami A, Javanmardi F, Pirbonyeh N, Akbari A (2020) Prevalence of underlying diseases in hospitalized patients with COVID-19: a systematic review and meta-analysis. Arch Acad Emerg Med 8(1):e35

38. Kaur N, Gupta I, Singh H et al (2020) Epidemiological and clinical characteristics of 6635 COVID-19 patients: a pooled analysis. SN Compr Clin Med. https://doi.org/10.1007/ s42399-020-00393-y

39. Hansrivijit P, Qian C, Boonpheng B et al (2020) Incidence of acute kidney injury and its association with mortality in patients with COVID-19: a meta-analysis. J Investig Med 68(7):1261-1270

40. Espinosa OA, Zanetti ADS, Antunes EF, Longhi FG, Matos TAd, Battaglini PF (2020) Prevalence of comorbidities in patients and mortality cases affected by SARS-CoV2: a systematic review and meta-analysis. Rev Inst Med Trop Sao Paulo 62:e43-e43

41. Baradaran A, Ebrahimzadeh MH, Baradaran A, Kachooei AR (2020) Prevalence of comorbidities in COVID-19 patients: a systematic review and meta-analysis. Arch Bone Jt Surg 8(Suppl 1):247-255

42. Goel S, Jain T, Hooda A et al (2020) Clinical characteristics and in-hospital mortality for COVID-19 across the globe. Cardiol Ther 9(2):553-559

43. Wang B, Luo Q, Zhang W et al (2021) The involvement of chronic kidney disease and acute kidney injury in disease severity and mortality in patients with COVID-19: a meta-analysis. Kidney Blood Press Res 46(1):17-30

44. Tisminetzky M, Delude C, Hebert T, Carr C, Goldberg RJ, Gurwitz JH (2020) Age, multiple chronic conditions, and COVID19: a literature review. J Gerontol A Biol Sci Med Sci. Dec 24:glaa320. https://doi.org/10.1093/gerona/glaa320. Epub ahead of print.

45. Farha MN, Md Momin I (2020) Prevalence of clinical manifestations and comorbidities of coronavirus (COVID-19) infection: a meta-analysis. Fortune J Health Sci. https://doi.org/10.26502/ fjhs009

46. Dorjee K, Kim H, Bonomo E, Dolma R (2020) Prevalence and predictors of death and severe disease in patients hospitalized due to COVID-19: A comprehensive systematic review and metaanalysis of 77 studies and 38,000 patients. PLoS ONE. https:// doi.org/10.1371/journal.pone.0243191

47. Rentsch CT, Kidwai-Khan F, Tate JP et al (2020) Covid-19 testing, hospital admission, and intensive care among 2,026,227 United States veterans aged 54-75 years. medRxiv 57:279

48. Ji W, Huh K, Kang M et al (2020) Effect of underlying comorbidities on the infection and severity of COVID-19 in Korea: a nationwide case-control study. J Korean Med Sci 35(25):e237

49. Corbett RW, Blakey S, Nitsch D et al (2020) Epidemiology of COVID-19 in an urban dialysis center. J Am Soc Nephrol 31(8):1815-1823

50. Fernandez Villalobos NV, Ott JJ, Klett-Tammen CJ et al (2020) Quantification of the association between predisposing health conditions, demographic, and behavioural factors with 
hospitalisation, intensive care unit admission, and death from COVID-19: a systematic review and meta-analysis. medRxiv 8:e001343

51. Wingert A, Pillay J, Gates M et al (2020) Risk factors for severe outcomes of COVID-19: a rapid review. medRxiv 38:5861

52. Chishinga N, Gandhi NR, Onwubiko UN, et al (2020) Characteristics and Risk Factors for Hospitalization and Mortality among Persons with COVID-19 in Atlanta Metropolitan Area. medRxiv 2020.2012.2015.20248214

53. Martos-Benitez FD, Soler-Morejon CD, Garcia-Del BD (2021) Chronic comorbidities and clinical outcomes in patients with and without COVID-19: a large population-based study using national administrative healthcare open data of Mexico. Intern Emerg Med. https://doi.org/10.1007/s11739-020-02597-5

54. Oetjens MT, Luo JZ, Chang A et al (2020) Electronic health record analysis identifies kidney disease as the leading risk factor for hospitalization in confirmed COVID-19 patients. PLoS ONE [Electronic Resource] 15(11):e0242182

55. Working group for the s, control of $\mathrm{C}$-iS, Members of the Working group for the s, control of C-iS (2020) The first wave of the COVID-19 pandemic in Spain: characterisation of cases and risk factors for severe outcomes, as at 27 April 2020. Euro Surveill 25(50): 12

56. Atkins JL, Masoli JAH, Delgado J et al (2020) Preexisting comorbidities predicting COVID-19 and mortality in the UK biobank community cohort. J Gerontol Ser A 75(11):2224-2230

57. Petrilli CM, Jones SA, Yang J et al (2020) Factors associated with hospital admission and critical illness among 5279 people with coronavirus disease 2019 in New York City: prospective cohort study. BMJ 369:m1966

58. Soares RdCM, Mattos LR, Raposo LM (2020) Risk factors for hospitalization and mortality due to COVID-19 in Espírito Santo State, Brazil. Am J Trop Med Hyg 103(3):1184-1190

59. Ko JY, Danielson ML, Town M et al (2020) Risk factors for COVID-19-associated hospitalization: COVID-19-associated hospitalization surveillance network and behavioral risk factor surveillance system. Clin Infect Dis 69:343

60. Ioannou GN, Locke E, Green P et al (2020) Risk factors for hospitalization, mechanical ventilation, or death among 10131 US veterans with SARS-CoV-2 Infection. JAMA Netw Open 3(9):e2022310-e2022310

61. Rossi PG, Marino M, Formisano D, Venturelli F, Vicentini M, Grilli R (2020) Characteristics and outcomes of a cohort of SARS-CoV-2 patients in the Province of Reggio Emilia, Italy. medRxiv. 2020:2020.2004.2013.20063545

62. Degarege A, Naveed Z, Kabayundo J, Brett-Major D (2020) Risk factors for severe illness and death in COVID-19: a systematic review and meta-analysis. MedRxiv. 323:2052

63. Cai R, Zhang J, Zhu Y, Liu L, Liu Y, He Q (2021) Mortality in chronic kidney disease patients with COVID-19: a systematic review and meta-analysis. Int Urol Nephrol. https://doi.org/10. 1007/s11255-020-02740-3

64. Zhou Y, Yang Q, Chi J, Dong B, Lv W, Shen L, Wang Y (2020) Comorbidities and the risk of severe or fatal outcomes associated with coronavirus disease 2019: A systematic review and metaanalysis. Int J Infect Dis 99:47-56. https://doi.org/10.1016/j.ijid. 2020.07.029. Epub 2020 Jul 25.

65. Islam MS, Barek MA, Aziz MA, Aka TD, Jakaria M (2020) Association of age, sex, comorbidities, and clinical symptoms with the severity and mortality of COVID-19 cases: a metaanalysis with 85 studies and 67299 cases. medRxiv. 16:1678

66. Izcovich A, Ragusa MA, Tortosa F et al (2020) Prognostic factors for severity and mortality in patients infected with COVID-19: A systematic review. PLoS ONE 121:62

67. Khan M, Khan MN, Mustagir MG, Rana J, Islam MS, Kabir MI (2020) Effects of underlying morbidities on the occurrence of deaths in COVID-19 patients: a systematic review and metaanalysis. medRxiv 26:26

68. Luo L, Fu M, Li Y et al (2020) The potential association between common comorbidities and severity and mortality of coronavirus disease 2019: a pooled analysis. Clin Cardiol. https://doi.org/10. $1002 /$ clc. 23465

69. Mehraeen E, Karimi A, Barzegary A et al (2020) Predictors of mortality in patients with COVID-19-a systematic review. Eur J Integr Med 40:101226-101226

70. Mesas AE, Cavero-Redondo I, Alvarez-Bueno C et al (2020) Predictors of in-hospital COVID-19 mortality: a comprehensive systematic review and meta-analysis exploring differences by age, sex and health conditions. PLoS ONE. https://doi.org/10. 1371/journal.pone.0241742

71. Hoo Jung Rhim, Jin Hyun Park, Yuna Lee et al (2020) Clinical, laboratory, and radiologic findings associated with mortality in COVID-19: a systematic review and meta-analysis. PREPRINT (Version 1) available at Research Square. https://doi.org/10. 21203/rs.3.rs-39877/v1

72. Sepandi M, Taghdir M, Alimohamadi Y, Afrashteh S, Hosamirudsari H (2020) Factors associated with mortality in Covid-19 patients a systematic review and meta-analysis. Iran J Public Health. https://doi.org/10.18502/ijph.v49i7.3574

73. Zhou S, Xu J, Xue C, Yang B, Mao Z, Ong ACM (2020) Coronavirus-associated kidney outcomes in COVID-19, SARS, and MERS: a meta-analysis and systematic review. Ren Fail. https:// doi.org/10.1080/0886022X.2020.1847724

74. Zhou X, Cheng Z, Shu D et al (2020) Characteristics of mortal COVID-19 cases compared to the survivors. Aging. https://doi. org/10.18632/aging.202216

75. Zhou Y, Ren Q, Chen G et al (2020) Chronic kidney diseases and acute kidney injury in patients with Covid-19: evidence from a meta-analysis. Front Med 7:588301

76. Biswas M, Rahaman S, Biswas TK, Haque Z, Ibrahim B (2020) association of sex, age, and comorbidities with mortality in COVID-19 patients: a systematic review and meta-analysis. Intervirology. https://doi.org/10.1159/000512592

77. Fang X, Li S, Yu H et al (2020) Epidemiological, comorbidity factors with severity and prognosis of COVID-19: a systematic review and meta-analysis. Aging 12(13):12493-12503

78. Noor FM, Islam MM (2020) Prevalence and associated risk factors of mortality among COVID-19 patients: a meta-analysis. J Community Health 45(6):1270-1282

79. Pranata R, Supriyadi R, Huang I et al (2020) The association between chronic kidney disease and new onset renal replacement therapy on the outcome of COVID-19 patients a meta-analysis. Clin Med Insights Circ Respir Pulm Med. https://doi.org/10. 1177/1179548420959165

80. Shi C, Wang L, Ye J, Gu Z, Wang S, Xia J, Xie Y, Li Q, Xu R, Lin N (2021) Predictors of mortality in patients with coronavirus disease 2019: a systematic review and meta-analysis. BMC Infect Dis 21(1):663

81. Ssentongo P, Ssentongo AE, Heilbrunn ES, Ba DM, Chinchilli VM (2020) Association of cardiovascular disease and 10 other pre-existing comorbidities with COVID-19 mortality: a systematic review and meta-analysis. PLoS ONE [Electronic Resource]. 15(8): 0238215

82. Chidambaram V et al (2020) Factors associated with disease severity and mortality among patients with COVID-19: A systematic review and meta-analysis. PLoS One 15(11):e0241541

83. Xiao W, Xu J, Liang X, Shi L, Zhang P, Wang Y, Yang H (2021) Relationship between chronic kidney disease and adverse outcomes of coronavirus disease 2019: a meta-analysis based on adjusted risk estimates. Int Urol Nephrol 53(8):1723-1727

84. Awortwe C, Cascorbi I (2020) Meta-analysis on outcomeworsening comorbidities of COVID-19 and related potential 
drug-drug interactions. Pharmacol Res. https://doi.org/10.1016/j. phrs.2020.105250

85. Bajgain KT, Badal S, Bajgain BB, Santana MJ (2020) Prevalence of comorbidities among individuals with COVID-19: a rapid review of current literature. Am J Infect Control. https://doi.org/ 10.1016/j.ajic.2020.06.21

86. Bajwa H, Riaz Y, Ammar M, Farooq S, Yousaf A (2020) The dilemma of renal involvement in COVID-19: a systematic review. Cureus. https://doi.org/10.7759/cureus.8632

87. Oyelade T, Alqahtani J, Canciani G (2020) Prognosis of COVID19 in patients with liver and kidney diseases: an early systematic review and meta-analysis. Trop Med Infect Dis. https://doi.org/ 10.3390/tropicalmed5020

88. Qiu P, Zhou Y, Wang F et al (2020) Clinical characteristics, laboratory outcome characteristics, comorbidities, and complications of related COVID-19 deceased: a systematic review and meta-analysis. Aging Clin Exp Res. https://doi.org/10.1007/ s40520-020-01664-3

89. Docherty AB, Harrison EM, Green CA et al (2020) Features of 20133 UK patients in hospital with covid-19 using the ISARIC WHO clinical characterisation protocol: prospective observational cohort study. BMJ 369:1985

90. Hewitt J, Carter B, Vilches-Moraga A et al (2020) The effect of frailty on survival in patients with COVID-19 (COPE): a multicentre, European, observational cohort study. Lancet Public Health 5(8):e444-e451

91. Murillo-Zamora E, Hernandez-Suarez CM (2021) Survival in adult inpatients with COVID-19. Public Health 190:1-3

92. Salinas-Escudero G, Carrillo-Vega MF, Granados-Garcia V, Martinez-Valverde S, Toledano-Toledano F, Garduno-Espinosa J (2020) A survival analysis of COVID-19 in the Mexican population. BMC Public Health 20(1):1616

93. Flythe JE, Assimon MM, Tugman MJ et al (2020) Characteristics and outcomes of individuals with pre-existing kidney disease and COVID-19 admitted to intensive care units in the United States. Am J Kidney Dis 77(2):190-203

94. Giorgi Rossi P, Marino M, Formisano D et al (2020) Characteristics and outcomes of a cohort of COVID-19 patients in the Province of Reggio Emilia Italy. PLoS ONE 15(8): $\mathrm{e} 0238281-\mathrm{e} 0238281$

95. Baqui P, Bica I, Marra V, Ercole A, van der Schaar M (2020) Ethnic and regional variations in hospital mortality from COVID-19 in Brazil: a cross-sectional observational study. Lancet Glob Health 8(8):e1018-e1026

96. Boulle A, Davies MA, Hussey H et al (2020) Risk factors for COVID-19 death in a population cohort study from the Western Cape Province South Africa. Clin Infect Dis 7:e314

97. Santos MM, Lucena EES, Lima KC, Brito AAC, Bay MB, Bonfada D (2019) Survival and predictors of deaths of patients hospitalized due to COVID-19 from a retrospective and multicenter cohort study in Brazil. Epidemiol Infect 194:119

98. Surendra H, Elyazar IR, Djaafara BA et al (2021) Clinical characteristics and mortality associated with COVID-19 in Jakarta, Indonesia: A hospital-based retrospective cohort study. Lancet Reg Health West Pac 9:100108

99. Grasselli G, Greco M, Zanella A et al (2020) Risk factors associated with mortality among patients with COVID-19 in intensive care units in Lombardy Italy. JAMA Intern Med 180(10):1345-1355

100. Portolés J, Marques M, López-Sánchez P et al (2020) Chronic kidney disease and acute kidney injury in the COVID-19 Spanish outbreak. Nephrol Dial Transplant 35(8):1353-1361

101. Ozturk S, Turgutalp K, Arici M et al (2020) Mortality analysis of COVID-19 infection in chronic kidney disease, haemodialysis and renal transplant patients compared with patients without kidney disease: a nationwide analysis from Turkey. Nephrol Dial Transplant 35(12):2083-2095

102. Lala A, Johnson KW, Januzzi JL et al (2020) Prevalence and impact of myocardial injury in patients hospitalized with COVID-19 infection. J Am Coll Cardiol 76(5):533-546

103. Kang SH, Kim SW, Kim AY, Cho KH, Park JW, Do JY (2020) Association between chronic kidney disease or acute kidney injury and clinical outcomes in COVID-19 patients. J Korean Med Sci 35(50):e434

104. Zandkarimi E, Moradi G, Mohsenpour B (2020) The prognostic factors affecting the survival of Kurdistan province COVID-19 patients: a cross-sectional study from February to May 2020. Int J Health Policy Manag. https://doi.org/10. 34172/ijhpm.2020.155

105. Klang E, Kassim G, Soffer S, Freeman R, Levin MA, Reich DL (2020) Severe obesity as an independent risk factor for COVID-19 mortality in hospitalized patients younger than 50 . Obesity (Silver Spring) 28(9):1595-1599

106. Macedo MCF, Pinheiro IM, Carvalho CJL et al (2020) Correlation between hospitalized patients' demographics, symptoms, comorbidities, and COVID-19 pandemic in Bahia, Brazil. PLoS ONE 15(12): 0243966

107. Lee SG, Park GU, Moon YR, Sung K (2020) Clinical characteristics and risk factors for fatality and severity in patients with coronavirus disease in korea: a nationwide population-based retrospective study using the korean health insurance review and assessment service (HIRA) database. Int J Environ Res Public Health 17(22): 18

108. Munblit D, Nekliudov NA, Bugaeva P et al (2020) StopCOVID cohort: An observational study of 3480 patients admitted to the Sechenov University hospital network in Moscow city for suspected COVID-19 infection. Clin Infect Dis. https://doi.org/10. 1093/cid/ciaa1535

109. Esme M, Koca M, Dikmeer A et al (2021) Older adults with coronavirus disease 2019: a nationwide study in Turkey. J Gerontol A Biol Sci Med Sci 76(3):e68-e75

110. Fried MW, Crawford JM, Mospan AR et al (2020) patient characteristics and outcomes of 11,721 patients with covid19 hospitalized across the United States. Clin Infect Dis. https://doi.org/10. 1093/cid/ciaa1268

111. Gude-Sampedro F, Fernández-Merino C, Ferreiro L et al (2021) Development and validation of a prognostic model based on comorbidities to predict COVID-19 severity: a population-based study. Int J Epidemiol 50(1):64-74

112. Harrison SL, Fazio-Eynullayeva E, Lane DA, Underhill P, Lip GYH (2020) Comorbidities associated with mortality in 31,461 adults with COVID-19 in the United States: a federated electronic medical record analysis. PLoS Med 17(9):e1003321

113. Jiménez E, Fontán-Vela M, Valencia J et al (2020) Characteristics, complications and outcomes among 1549 patients hospitalised with COVID-19 in a secondary hospital in Madrid, Spain: a retrospective case series study. BMJ Open 10(11):e042398

114. Kaeuffer C, Le Hyaric C, Fabacher T et al (2020) Clinical characteristics and risk factors associated with severe COVID-19: prospective analysis of 1045 hospitalised cases in North-Eastern France, March 2020. Euro Surveill. https://doi.org/10.2807/15607917.ES.2020.25.48.2000895

115. Rapp JL, Lieberman-Cribbin W, Tuminello S, Taioli E (2021) Male sex, severe obesity, older age, and chronic kidney disease are associated with COVID-19 severity and mortality in New York City. Chest 159(1):112-115

116. Kim DW, Byeon KH, Kim J, Cho KD, Lee N (2020) The correlation of comorbidities on the mortality in patients with COVID19: an observational study based on the Korean National Health insurance big data. J Korean Med Sci 35(26):e243 
117. Imam Z, Odish F, Gill I et al (2020) Older age and comorbidity are independent mortality predictors in a large cohort of 1305 COVID-19 patients in Michigan United States. J Intern Med 288(4):469-476

118. Panagiotou OA, Kosar CM, White EM et al (2021) risk factors associated with all-cause 30-day mortality in nursing home residents with COVID-19. JAMA Intern Med. https://doi.org/10. 1001/jamainternmed.2020.7968

119. Almazeedi S, Al-Youha S, Jamal MH et al (2020) Characteristics, risk factors and outcomes among the first consecutive 1096 patients diagnosed with COVID-19 in Kuwait. EClinicalMedicine. 24:100448

120. de Souza CD, de Arruda Magalhães AJ, Lima AJ et al (2020) Clinical manifestations and factors associated with mortality from COVID-19 in older adults: Retrospective population-based study with 9807 older Brazilian COVID-19 patients. Geriatr Gerontol Int 20(12):1177-1181

121. Parra-Bracamonte GM, Parra-Bracamonte FE, Lopez-Villalobos N, Lara-Rivera AL (2021) Chronic kidney disease is a very significant comorbidity for high risk of death in patients with COVID-19 in Mexico. Nephrology (Carlton) 26(3):248-251

122. Akchurin O, Meza K, Biswas S et al (2021) COVID-19 in Patients with CKD in New York City. Kidney360 2(1):63

123. Ng JH, Hirsch JS, Wanchoo R et al (2020) Outcomes of patients with end-stage kidney disease hospitalized with COVID-19. Kidney Int 98(6): 1530-1539

124. Villar-García J, Vivanco-Hidalgo RM, Clèries M et al (2020) Risk factors for SARS-CoV-2 infection, hospitalisation, and death in Catalonia, Spain: a population-based cross-sectional study. medRxiv 9:1548

125. Dominguez-Ramirez L, Rodriguez-Perez F, Sosa-Jurado F, Santos-Lopez G, Cortes-Hernandez P (2020) The role of metabolic comorbidity in COVID-19 mortality of middle-aged adults. The case of Mexico. medRxiv. 69:343

126. Kim L, Garg S, O'Halloran A et al (2020) Risk factors for intensive care unit admission and in-hospital mortality among hospitalized adults identified through the US Coronavirus Disease 2019 (COVID-19)-associated hospitalization surveillance network (COVID-NET). Clin Infect Dis. https://doi.org/10.1093/ cid/ciaa1012

127. Barek MA, Aziz MA, Islam MS (2020) Impact of age, sex, comorbidities and clinical symptoms on the severity of COVID19 cases: a meta-analysis with 55 studies and 10014 cases. Heliyon. 6(12):e05684

128. Henry BM, Lippi G (2020) Chronic kidney disease is associated with severe coronavirus disease 2019 (COVID-19) infection. Int Urol Nephrol 52(6):1193-1194

129. Izcovich A, Ragusa MA, Tortosa F et al (2020) Prognostic factors for severity and mortality in patients infected with COVID-19: a systematic review. PLoS ONE 15(11):e0241955

130. Li J, He X, Yuan Y et al (2021) Meta-analysis investigating the relationship between clinical features, outcomes, and severity of severe acute respiratory syndrome coronavirus 2 (SARS-CoV-2) pneumonia. Am J Infect Control 49(1):82-89

131. Luo L, Fu M, Li Y et al (2020) The potential association between common comorbidities and severity and mortality of coronavirus disease 2019: a pooled analysis. Clin Cardiol 43(12):1478-1493

132. Mudatsir M, Fajar JK, Wulandari L et al (2020) Predictors of COVID-19 severity: a systematic review and meta-analysis. F1000Res 9:1107

133. Plasencia-Urizarri TM, Aguilera-Rodríguez R, AlmaguerMederos LE (2020) Comorbidities and clinical severity of COVID-19: systematic review and meta-analysis. Rev habanera cienc méd. 19(supl.1):e3389-e3389
134. Radwan NM, Mahmoud NE, Alfaifi AH, Alabdulkareem KI (2020) Comorbidities and severity of coronavirus disease 2019 patients. Saudi Med J 41(11):1165-1174

135. Tabrizi R, Lankarani KB, Nowrouzi-sohrabi P et al (2020) The role of comorbidities and clinical predictors of severe disease in COVID-19: a systematic review and meta-analysis. medRxiv. 395:1225

136. Toraih EA, Elshazli RM, Hussein MH et al (2020) Association of cardiac biomarkers and comorbidities with increased mortality, severity, and cardiac injury in COVID-19 patients: a meta-regression and decision tree analysis. J Med Virol 92(11):2473-2488

137. Wang B, Li R, Lu Z, Huang Y (2020) Does comorbidity increase the risk of patients with COVID-19: evidence from meta-analysis. Aging (Albany NY) 12(7):6049-6057

138. Wang X, Fang X, Cai Z et al (2020) Comorbid chronic diseases and acute organ injuries are strongly correlated with disease severity and mortality among COVID-19 patients: a systemic review and meta-analysis. Research (Wash D C) 2020:2402961

139. Yanling $\mathrm{Wu}, \mathrm{Hu} \mathrm{Li}$, Shengjin Li et al (2020) Clinical determinants of the severity of Coronavirus disease 2019 (COVID-19): a systematic review and meta-analysis. PREPRINT (Version 1) available at Research Square [https://doi.org/10.21203/rs.3.rs$56852 / \mathrm{v} 1]$

140. Liu YF, Zhang Z, Pan XL et al (2021) The chronic kidney disease and acute kidney injury involvement in COVID-19 pandemic: a systematic review and meta-analysis. PLoS ONE 16(1):e0244779

141. Zaki N, Mohamed EA, Ibrahim S, Khan G (2020) The influence of comorbidity on the severity of COVID-19 disease: a systematic review and analysis. medRxiv. 2020:2020.2006.2018.20134478

142. Awortwe C, Cascorbi I (2020) Meta-analysis on outcome-worsening comorbidities of COVID-19 and related potential drugdrug interactions. Pharmacol Res 161:105250

143. Fang X, Li S, Yu H et al (2020) Epidemiological, comorbidity factors with severity and prognosis of COVID-19: a systematic review and meta-analysis. Aging (Albany NY) 12(13):12493-12503

144. Pranata R, Supriyadi R, Huang I et al (2020) The association between chronic kidney disease and new onset renal replacement therapy on the outcome of covid-19 patients: a meta-analysis. Clin Med Insights Circ Respir Pulm Med 14:1179548420959165

145. Liu D, Cui P, Zeng S, Wang S, Feng X, Xu S, Li R, Gao Y, Yu R, Wang Y, Yuan Y (2020) Risk factors for developing into critical COVID-19 patients in Wuhan, China: a multicenter, retrospective, cohort study. Eclinicalmedicine 25:100471

146. Kim SR, Nam SH, Kim YR (2020) Risk factors on the progression to clinical outcomes of Covid-19 patients in south korea: using national data. Int J Environ Res Public Health. https://doi. org/10.3390/ijerph 17238847

147. Omrani AS, Almaslamani MA, Daghfal J, Alattar RA, Elgara M, Shaar SH, Ibrahim TB, Zaqout A, Bakdach D, Akkari AM, Baiou A (2020) The first consecutive 5000 patients with Coronavirus Disease 2019 from Qatar; a nation-wide cohort study. BMC Infect Dis 20(1):777

148. Fried MW, Crawford JM, Mospan AR, Watkins SE, Munoz B, Zink RC, Elliott S, Burleson K, Landis C, Reddy KR, Brown RS $\mathrm{Jr}$ (2020) Patient characteristics and outcomes of 11,721 patients with Covid19 hospitalized across the United States. Clin Infect Dis 2(10):e558-e565

149. Bellou V, Tzoulaki I, Evangelou E, Belbasis L (2020) Risk factors for adverse clinical outcomes in patients with COVID-19: asystematic review and meta-analysis. medRxiv. https://doi.org/ 10.1101/2020.05.13.20100495

150. Imam Z, Odish F, Gill I et al (2020) Older age and comorbidity are independent mortality predictors in a large cohort of 1305 COVID-19 patients in Michigan, United States. J Intern Med 288(4):469-476 
151. Saran R, Robinson B, Abbott KC et al (2020) US renal data system 2019 annual data report: epidemiology of kidney disease in the United States. Am J Kidney Dis 75(1 Suppl 1):A6-a7

152. Sarnak MJ, Jaber BL (2000) Mortality caused by sepsis in patients with end-stage renal disease compared with the general population. Kidney Int 58(4):1758-1764

153. Naqvi SB, Collins AJ (2006) Infectious complications in chronic kidney disease. Adv Chronic Kidney Dis 13(3):199-204

154. Sarnak MJ, Jaber BL (2001) Pulmonary infectious mortality among patients with end-stage renal disease. Chest 120(6): 1883-1887

155. Kato S, Chmielewski M, Honda $\mathrm{H}$ et al (2008) Aspects of immune dysfunction in end-stage renal disease. Clin J Am Soc Nephrol 3(5):1526-1533

156. de Almeida-Pititto B, Dualib PM, Zajdenverg L et al (2020) Severity and mortality of COVID 19 in patients with diabetes, hypertension and cardiovascular disease: a meta-analysis. Diabetol Metab Syndr 12:75
157. Salman B, Hussain M, Shafique K, Imtiaz S, Dhrolia MF (2018) Risk factors of hospitalization among chronic kidney disease patients in tertiary care hospitals-a single-center experience. Saudi J Kidney Dis Transpl 29(5):1150-1158

158. Agur T, Ben-Dor N, Goldman S et al (2021) Antibody response to mRNA SARS-CoV-2 vaccine among dialysis patients-a prospectivecohort study. Nephrol Dial Transplant. https://doi.org/10. 1093/ndt/gfab155

159. Grupper A, Sharon N, Finn T et al (2021) Humoral RESPONSE TO THE Pfizer BNT162b2 vaccine in patients undergoing maintenance hemodialysis. Clin J Am Soc Nephrol. https://doi.org/10. 2215/CJN.03500321

Publisher's Note Springer Nature remains neutral with regard to jurisdictional claims in published maps and institutional affiliations. 